The following paper posted here is not the official IEEE published version. The final published version of this paper can be found in the Proceedings of the IEEE Industry Applications Conference. Annual Meeting. (41st : 2006 : Tampa, Florida):pp.1962-1969

Copyright @ 2006 IEEE.

Personal use of this material is permitted. However, permission to reprint/republish this material for advertising or promotional purposes or for creating new collective works for resale or redistribution to servers or lists, or to reuse any copyrighted component of this work in other works must be obtained from the IEEE. 
The following paper posted here is not the official IEEE published version. The final published version of this paper can be found in the Proceedings of the IEEE Industry Applications Conference. Annual Meeting. (41st : 2006 : Tampa, Florida):pp.1962-1969

Copyright @ 2006 IEEE.

Personal use of this material is permitted. However, permission to reprint/republish this material for advertising or promotional purposes or for creating new collective works for resale or redistribution to servers or lists, or to reuse any copyrighted component of this work in other works must be obtained from the IEEE. 


\section{Impact of Maximum Back-EMF Limits on the Performance Characteristics of Interior Permanent Magnet Synchronous Machines}

\author{
Seok-Hee Han \\ Thomas M. Jahns \\ Department of Electrical and Computer Engineering \\ University of Wisconsin - Madison \\ Madison, WI \\ seokheehan@wisc.edu $\quad$ jahns@engr.wisc.edu
}

\author{
Metin Aydin \\ Department of Mechatronics Engineering \\ Kocaeli University \\ Veziroglu Campus, 41040 Kocaeli, TURKEY \\ maydin@ieee.org
}

\author{
Mustafa K. Guven \\ Caterpillar Inc. \\ Tech Center \\ Peoria, IL \\ Guven_Mustafa_K@,cat.com
}

\author{
Wen L. Soong \\ School of Electrical and Electronic Engineering \\ University of Adelaide \\ Adelaide, Australia \\ wlsoong@eleceng.adelaide.edu.au
}

\begin{abstract}
Interior permanent magnet (IPM) synchronous machines are vulnerable to uncontrolled generator (UCG) faults at high speed that can damage the inverter. One approach to reducing this risk is to impose limits on the maximum machine back-emf voltage at top speed. This paper presents the results of a comparative design study that clarifies the nature and extent of the penalties imposed on the IPM machine metrics and performance characteristics as a result of imposing progressively tighter values of back-emf voltage limits. As an alternative to limiting back-emf and penalizing machine designs, this paper also investigates the effectiveness of the system-side protection approach to the same UCG fault problem.
\end{abstract}

Keywords- interior permanent magnet synchronous machine; IPM, uncontrolled generator faults; UCG; back-emf limit

\section{INTRODUCTION AND PAPER PURPOSE}

Both interior permanent magnet (IPM) and surface permanent magnet (SPM) synchronous machines are receiving considerable attention as candidates for applications that require wide speed ranges of constant power operation [1]. However, one of the serious problems that constrains the application of PM synchronous machines in applications requiring high constant-power speed ratio (CPSR) values is the fault mode known as uncontrolled generator (UCG) operation [3].

The UCG operating mode is triggered if a fault condition results in the removal of gating from the inverter switches when the rotor is spinning at high speed (see Fig. 1). Under these conditions, the free-wheeling diodes in the inverter act as a three-phase uncontrolled rectifier to deliver braking/generator current back to the dc bus. If the dc bus is not capable of absorbing this energy using well-known techniques such as regenerative inversion back to the ac utility source, the dc bus capacitance will quickly charge up to the peak line-to-line

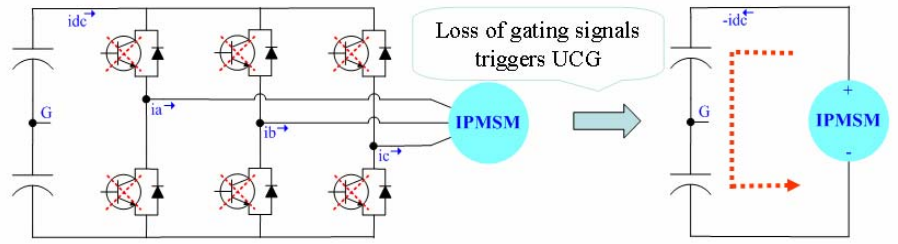

Figure 1. Uncontrolled generator (UCG) fault mode operation

machine back-emf voltage. Worst-case conditions occur when the machine is operating at its top speed, causing the back-emf voltages to reach their maximum amplitudes. For high CPSR applications, the maximum line-to-line back-emf amplitude can be several times higher than the nominal dc link voltage, exposing the dc bus capacitors and the inverter power switches to dangerously high overvoltages that are seriously destructive.

In this paper, two approaches for mitigating this risk of dangerously high back-emf voltages during UCG fault mode operation are investigated. One approach is based on modifying the machine design by imposing limits on the maximum backemf voltage of the machine at top speed. This approach has the advantage of preventing the overvoltage problem from ever occurring by constraining the back-emf voltage source amplitude. However, the required machine modifications introduce performance penalties that must be considered.

The second approach to the same problem is the introduction of robust drive system protection that prevents any dangerous overvoltage from appearing on the dc bus. Accordingly, any incipient overvoltage condition is quickly detected, causing a crowbar circuit to either clamp or short-circuit the dc bus terminals. In the process, the three machine terminals are also simultaneously clamped or short-circuited, resulting in machine phase currents that are safely limited (for steady-state fault mode operation) to the machine's characteristic current amplitude $\left(I_{c h}=\lambda_{P M} / L_{d}\right)[3]$. 
IPM synchronous machines can better cope with a maximum limit placed on the back-emf voltage at top speed than SPM machines because of the availability of reluctance torque in IPM machines. That is, the strength of the rotor magnets in IPM machines can be reduced compared to the magnets in the comparably-rated SPM machine by designing the IPM machine to make heavier use of reluctance torque [2].

However, the introduction of this additional constraint can have a significant negative impact on the metrics (e.g., mass, volume) and the performance of the resulting IPM machine design. There is little available in the literature that directly addresses the IPM machine design tradeoffs that are produced by constraining the maximum amplitude of the back-emf at top speed. The impact of the back-emf constraint on inverter current rating was addressed briefly on a theoretical basis in [4] without directly addressing the realizability of the resulting machine designs. The issue was raised again in a more recent paper [5] that compares the designs of two IPM machines for the same set of specifications for which the only difference is the imposition of a tight maximum back-emf constraint for one of the two designs.

This paper has two objectives. The first objective is to thoroughly investigate the impact of maximum back-emf constraints on the resulting design metrics and performance characteristics of IPM synchronous machines. Although it is necessary to limit this investigation to a particular class of IPM machines, key results can be generalized to a broader range of IPM machine designs.

The second objective is to explore the use of inverter bus overvoltage protection as an alternative means of preventing damage from UCG faults in IPM machine drives without resorting to constraints on the machine back-emf amplitude. The transient response of the drive system to a UCG fault followed by the sudden activation of a dc bus crowbar circuit is simulated to explore the resulting electrical stresses on the inverter and machine.

Section II describes the study conditions and IPM machine specification used to perform this investigation, followed by a summary of the IPM machine design tool features. Section III presents the major study results describing the impact of IPM machine back-emf constraints on performance metrics. Next, Section IV investigates the effectiveness of the alternative system-level approach to addressing the back-emf amplitude issue using dc bus overvoltage protection. Finally, Section V summarizes the major conclusions.

\section{Study Conditions And Machine Design ToOL}

\section{A. Adopted Per-Unit System and Machine Specifications}

The investigation has been carried out on a particular IPM machine which has a power rating in excess of $100 \mathrm{~kW}$. This machine is designed for an electric traction application which requires a challenging constant power speed ratio of $6: 1$. The maximum allowable stator current density used has been set at $10 \mathrm{~A}_{\mathrm{rms}} / \mathrm{mm}^{2}$ which is consistent with an assumption of liquid cooling.

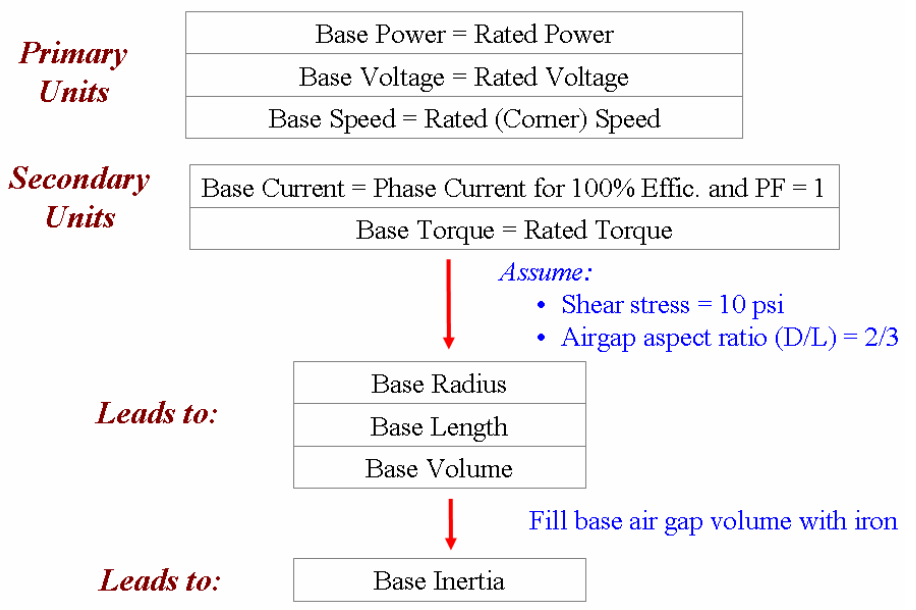

Figure 2. Basis for adopted per-unit system

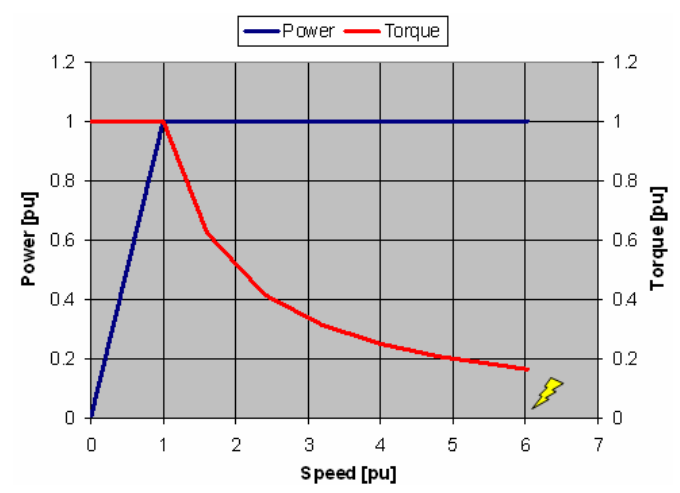

Figure 3. Normalized machine output power specification

In order to generalize the results of this study to the greatest possible extent, a normalized per-unit system is used for the presentation of all key results. Figure 2 provides a summary of the primary and secondary base units that have been adopted.

The primary base units are the typical choices, consisting of rated power, rated line-to-line rms voltage, and rated (i.e., corner) speed. Derived secondary base units, consistent with common practice, include base current and base torque as expressed in Fig. 2.

In addition to these rather conventional base units, this perunit system also develops base units for radius and length dimensions that are derived by adopting reference values for the rotor aspect ratio and the airgap shear stress, as summarized in Fig. 2. This, in turn, makes it possible to define a base rotor moment of inertia corresponding to a solid iron cylindrical mass with radial and length dimensions of 1 pu. Finally, it is convenient to define a per-unit value for back-emf voltage as the ratio of the peak line-to-line back-emf voltage at top speed to the nominal de link voltage.

The IPM machine output power specification is summarized in Fig. 3. As indicated in this figure, all candidate machines are required to deliver 1 pu output power from the corner speed of $1 \mathrm{pu}$ to a maximum speed of $6 \mathrm{pu}$, corresponding to a CPSR value of 6 . The maximum available terminal voltage for all candidate machines at any speed is $1 \mathrm{pu}$. 


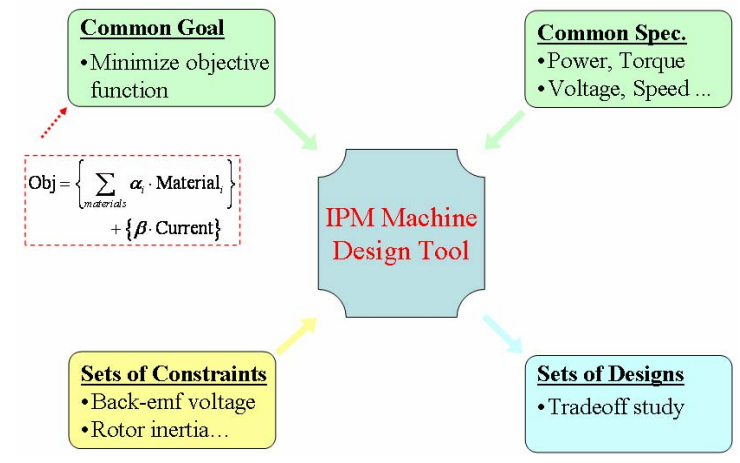

Figure 4. Overview of IPM machine design approach

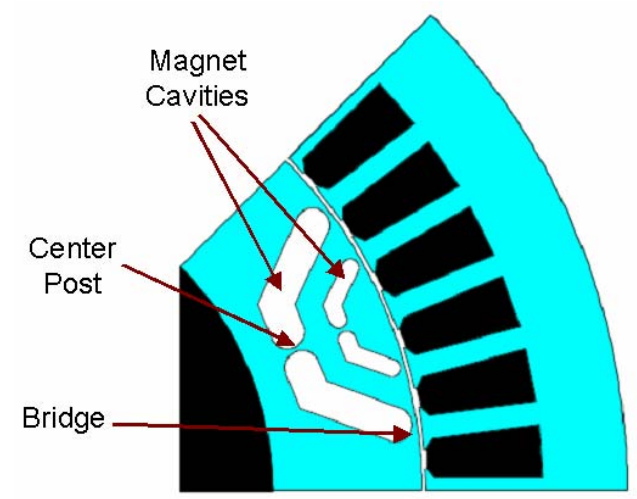

Figure 5. Cross-section of IPM machine configuration used in study

\section{B. Investigation Analytical Approach}

The analytical approach used to carry out this investigation is summarized in Fig. 4. A series of optimized IPM machine designs are developed using a numerical IPM machine design tool that seeks to minimize a scalar objective function. The objective function in this study is a weighted average of material mass approximating the machine material cost plus an additional weighted term proportional to the machine rated current, reflecting inverter cost and weight. Mathematically, this objective function can be expressed as

$$
\text { Objective }=\sum_{\text {materials }} \alpha_{i} \cdot \text { mass }_{i}+\beta \cdot \text { Current }
$$

where the values of the weighting constants $\alpha_{i}$ are selected to represent (approximately) the relative costs of the three major machine materials (copper, iron, and $\mathrm{NdFeB}$ magnets) and the value of weighting constant $\beta$ is selected to reflect the incremental cost of increasing the inverter's current rating.

All of the IPM machines are subjected to the same performance specifications for power, torque, voltage, and speed. In addition to these shared requirements, limits on the maximum back-emf voltage at top speed and maximum rotor moment of inertia are adjusted in order to develop several sets of optimized IPM machine designs that meet different combinations of these constraints. Performance metrics of these alternative machine designs that have been developed subject to different back-emf and moment of inertia constraints are then compared to examine the impacts of these limits.

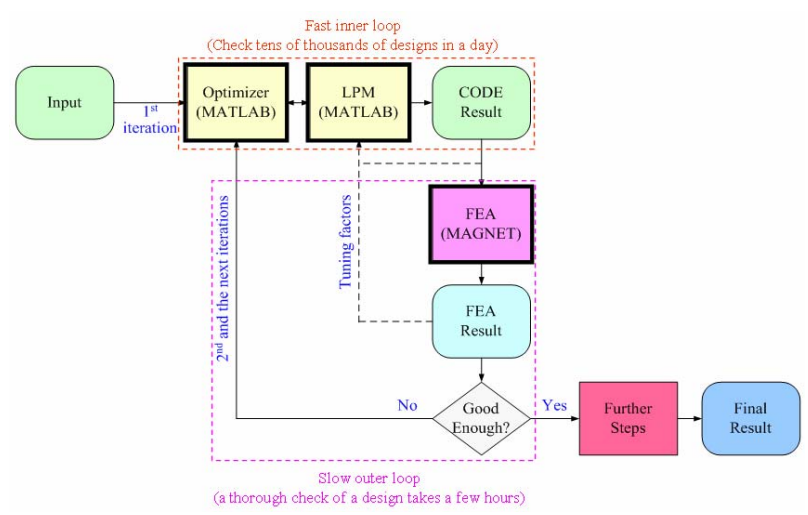

Figure 6. Basic flow diagram for IPM machine design optimization

TABLE I. MINIMUM AND MAXIMUM RANGES OF KEY MACHINE DESIGN PARAMETERS USED IN THE OPTIMIZATION

\begin{tabular}{|l|c|c|}
\hline Parameter & Minimum & Maximum \\
\hline Magnet remanent flux density & $0.25 \mathrm{~T}$ & $1.05 \mathrm{~T}$ \\
\hline Number of poles & 4 & 8 \\
\hline Stator outer radius & $1.37 \mathrm{pu}$ & $2.36 \mathrm{pu}$ \\
\hline Active stack length & $0.92 \mathrm{pu}$ & $1.60 \mathrm{pu}$ \\
\hline Ratio of rotor OD to stator OD & 0.4 & 0.7 \\
\hline Ratio of rotor ID to rotor OD & 0.5 & 0.82 \\
\hline $\begin{array}{l}\text { Ratio of total magnet thickness to } \\
\text { rotor width [=(rotor OD-rotor ID)/2] }\end{array}$ & 0.2 & 0.5 \\
\hline
\end{tabular}

The scope of this investigation has been limited to IPM machine rotor designs with two magnet layers in each rotor pole (see Fig. 5). This machine uses single-piece rotor laminations with narrow steel bridges and posts. The study was also limited to distributed three phase stator windings with two slots per pole per phase. The stator and rotor dimensions, magnet remanent flux density, and number of poles are free design variables. The allowable ranges of some of the free design variables are shown in Table I.

The IPM machine design tool shown in Fig. 6 consists of three components: a genetic optimizer, a lumped-parameter magnetic circuit model of the IPM machine, and a finite element analysis (FEA) tool used for verification and model tuning. The analytical lumped-parameter magnetic circuit model for IPM machines presented in [6] has been combined with differential evolution, a relatively recent addition to the family of genetic algorithms [7], to perform the machine design optimization. The differential evolution optimizer combined with the lumped parameter model (LPM) is capable of efficiently searching vast multi-dimensional spaces of free design variables under demanding constraints and competing objectives. The FEA verification and model tuning tools serves as a slow outer loop to compensate for any inaccuracies in the lumped parameter model. This combination of LPM and FEA provides an effective engineering compromise between optimization speed and accuracy.

Using the same machine specifications and design objective function described above, the two design constraints for maximum back-emf voltage $V_{\text {bemf-max }}$ and rotor moment of inertia $J_{\max }$ are adjusted to develop six optimized IPM machine designs illustrated in Fig. 7. First, the value $V_{\text {bemf-max }}$ is 


\begin{tabular}{|c|c|c|c|c|c|c|}
\hline & $\int_{0}^{A}$ & $\widehat{V}^{\mathrm{B}}$ & $\begin{array}{l}\vee^{\mathbf{C}} \\
\odot\end{array}$ & $\begin{array}{l}\curvearrowright^{\mathrm{D}} \\
\odot \\
\odot\end{array}$ & $\begin{array}{l}\approx^{\mathbf{E}} \\
\text { (?) }\end{array}$ & $\approx^{\mathrm{F}}$ \\
\hline $\begin{array}{c}\text { Back-emf } \\
\text { [pu] }\end{array}$ & 3.6 & 2.7 & 2.3 & 1.8 & 1.8 & 1.8 \\
\hline $\begin{array}{c}\text { Inertia } \\
{[\mathrm{pu}]}\end{array}$ & 1.1 & 1.1 & 1.1 & 1.1 & 1.7 & $\infty$ \\
\hline
\end{tabular}

Figure 7. Six sets of IPM machine design constraints used to investigate impact of back-emf and moment of inertia limits

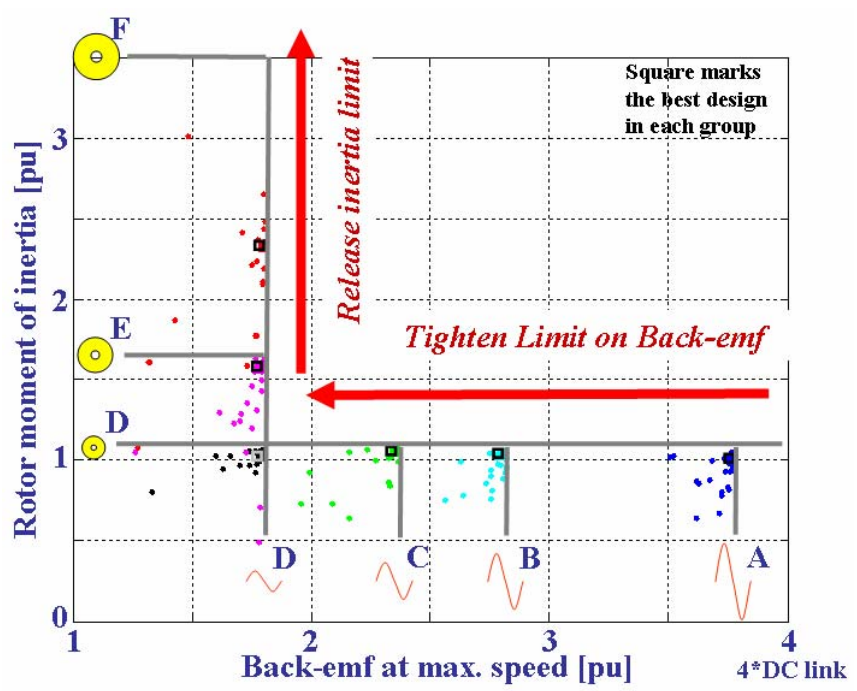

Figure 8. Six optimized machine design groups resulting from application of design constraints defined in Fig. 7

progressively reduced in steps from 3.6 pu to 1.8 pu to produce a group of four optimized machine designs while holding $J_{\max }$ constant at $1.1 \mathrm{pu}$. Next, two additional designs are developed using progressively higher values of $J_{\max }$ while holding the back-emf limit $V_{\text {bemf-max }}$ fixed at $1.8 \mathrm{pu}$. The value of $1.8 \mathrm{pu}$ was chosen for the lowest value of $V_{\text {bemf-max }}$ based on the maximum voltage ratings of the power semiconductors in the inverter. Choosing a lower value of $V_{\text {bemf-max }}$ would not change any of the major trends or conclusions of this study.

To assist reader with understanding the progression of these stepped changes to $V_{\text {bemf-max }}$ and $J_{\max }$, the relative amplitudes of these limits are represented by the sizes of the sine wave and rotor disk that appears in the upper row of Fig. 7. In addition, letters $\mathrm{A}$ to $\mathrm{F}$ are also assigned to the six sequential sets of design constraints. The resulting performance metric comparisons among the six resulting machine designs $\mathrm{A}$ to $\mathrm{F}$ are presented in the next section.

\section{COMParison OF CONSTRaint CASE Results}

The IPM machine design tool discussion in Section II was used to develop 6 optimized machine designs, one for each of the constraint cases designated A to F. Figure 8 captures a subset of the most promising machine designs for each of these constraint cases on a two-dimensional plot with maximum back-emf at top speed along the $x$-axis and rotor moment of inertia along the $y$-axis. In the figure, each dot represents a specific machine design.
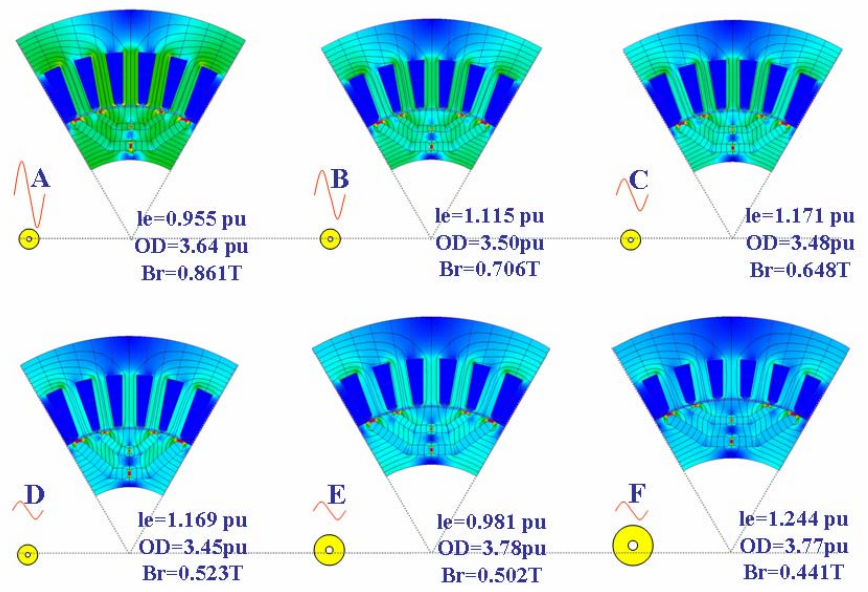

Figure 9. Cross sections of the six optimized IPM machine designs

Each of the constraint cases A to F results in a separate, identifiable group of candidate machine designs. The design that yields the lowest value of the objective function in each group, corresponding to the optimum machine design for that particular constraint case, is designated with a square.

It can be observed that, with the exception of case F, the best machine design for each case takes advantage of the maximum back-emf $V_{\text {bemf-max }}$ and the maximum moment of inertia $J_{\max }$ that are permissible for that case. In case $\mathrm{F}$, the rotor moment of inertia optimizes at value in the vicinity of 2.3 pu when the $J_{\max }$ limit is raised to infinity.

All results presented in this section are presented in a consistent fashion proceeding from cases $A$ to $F$. That is, the back-emf limit $V_{\text {bemf-max }}$ is gradually reduced while maintaining the lowest moment of inertia limit $J_{\max }$ in order to explore the resulting penalty on the performance metrics of the optimized machine for cases A through D. Next, the limit on the rotor moment of inertia $J_{\max }$ is gradually raised while maintaining the lowest back-emf limit (Cases D through F), in order to explore any available recovery in the performance metric values from the penalties suffered by lowering $V_{\text {bemf-max }}$.

Figure 9 presents single-pole cross-sections of the six best machine designs for cases $\mathrm{A}$ through $\mathrm{F}$ together with the active stack length (le), stator outer diameter $(O D)$, and magnet remanent flux density $(B r)$. It should be noted that the machine diameter and volume change little for cases $\mathrm{A}$ through $\mathrm{D}$ for which the value of $J_{\max }$ is constant, but then increase for cases $\mathrm{E}$ and $\mathrm{F}$ due to the increasing $J_{\max }$ limit value. In contrast, the value of the magnet remanent flux density $B r$ decreases consistently from cases A through $\mathrm{F}$ as $V_{\text {bemf-max }}$ is first reduced and $J_{\max }$ is subsequently increased.

Figure 10 provides a normalized comparison of the value of the objective function (1) achieved for each of the 6 constraint cases. Since the objective function was defined to reflect machine and inverter cost, the lowest possible value of the objective function is desired. The results in Fig. 10 show that there is a penalty of approx. $34 \%$ in the objective function value as the back-emf voltage limit $V_{\text {bemf-max }}$ is reduced from 3.6 pu (Case A) to $1.8 \mathrm{pu}$ (Case D). By subsequently releasing the moment of inertia constraint, a significant portion of this 


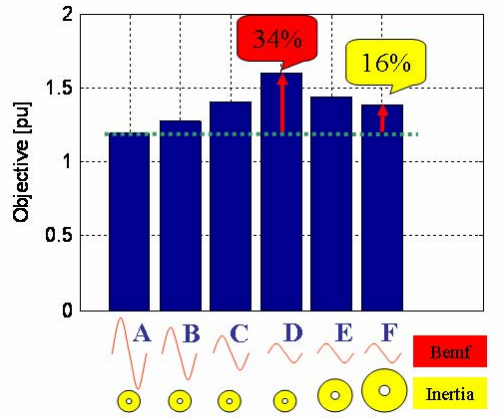

Figure 10. Objective function evaluation for optimized IPM machine designs for six sets of back-emf and moment of inertia constraints
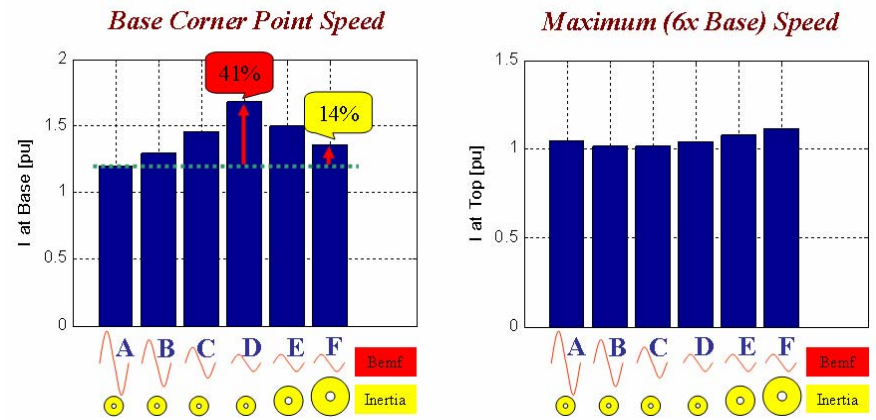

Figure 11. Calculated current amplitude comparisons among the six optimized IPM machine designs at rated output power evaluated at base speed (left) and maximum speed (right)

penalty is recovered. However, the resulting objective function value for Case F with $J_{\max }$ raised to infinity is still $16 \%$ higher than the corresponding value for Case A with $V_{\text {bemf-max }}$ set at its highest value of $3.6 \mathrm{pu}$.

Figure 11 plots the impact of the $V_{\text {bemf-max }}$ and $J_{\max }$ limits on the phase current requirements at the corner speed (Fig. 11a) and top speed (Fig. 11b). It is clearly shown that there is a significant penalty in higher required phase current at the corner speed caused by reducing the back-emf voltage limit (i.e., $41 \%$ increase for Case D compared to Case A). However, similarly to the trend in Fig. 10, approx. two-thirds of this penalty at the corner speed can be recovered by accepting a significantly higher rotor moment of inertia (Cases D to F).

In contrast, it is interesting to note in Fig. $11 \mathrm{~b}$ that the required phase current at top speed (6 pu) is affected very little by the values of $V_{\text {bemf-max }}$ or $J_{\max }$. This can be explained by the fact that the best machine designs for the six constraint cases converge to the value of the characteristic current $I_{c h}$ at top speed, and the value of $I_{c h}$ will be in the vicinity of 1 pu for all six cases.

Figure 12 provides more insights into the impact of the $V_{\text {bemf-max }}$ and $J_{\max }$ constraints on the machine's rated current at the corner speed and machine volume by comparing the results for three of the key constraint cases (Cases A, D, and F). More specifically, the best machine designs for Cases A, D, and F are plotted as points in the plane defined by the rated current at the corner point speed ( $x$-axis) and the machine stator volume (yaxis). Here again, each dot in the two-dimensional plot represents a specific design and squares again represent the best designs for the three cases considered.

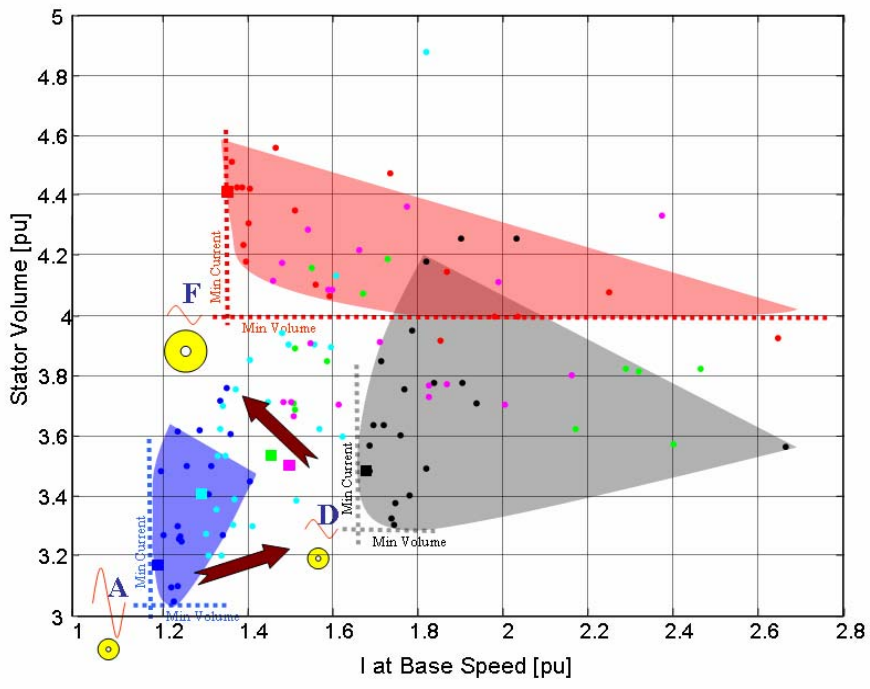

Figure 12. Illustration of tradeoffs between rated current and machine volume for IPM machines designed with different sets of back-emf and moment of inertia constraints. Squares represent best machine in each constraint group.

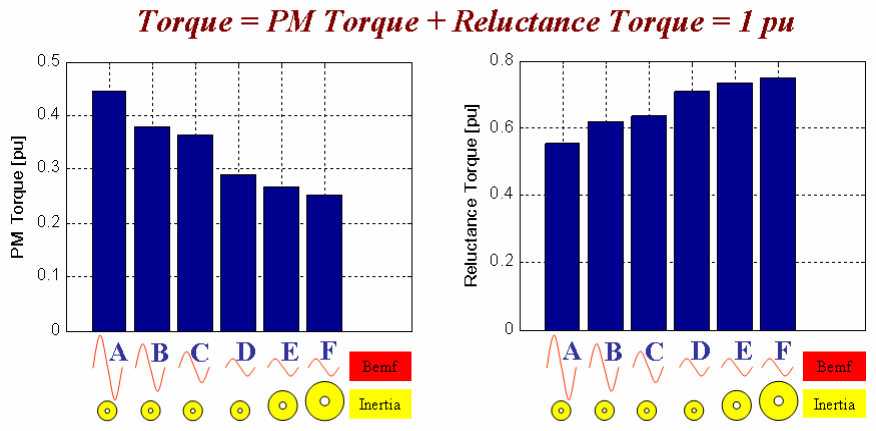

Figure 13. Torque component breakdown at rated speed for the six optimized IPM machine designs: PM torque component (left) and reluctance torque component (right)

In Fig. 12, the best possible design corresponds to the lower left corner (the smallest inverter current rating and motor size), while the worst design corresponds to the upper right corner. Consistent with the results in Figs. 10 and 11, the designs corresponding to Case A provide the best current and volume metrics among all of the 6 cases, appearing close to the lower left corner of the plot.

When the back-emf limit $V_{\text {bemf-max }}$ is reduced from $3.6 \mathrm{pu}$ (Case A) to $1.8 \mathrm{pu}$ (Case D), the resulting machine designs shift to significantly higher values of rated current and modestly higher stator volume values. When the rotor moment of inertia limit $J_{\max }$ is released while the back-emf limit is held at $1.8 \mathrm{pu}$ (Case F), the rated current is significantly reduced (i.e., shifted leftward), and the penalty is transferred to the stator volume which increases quite noticeably. The resulting tradeoffs that exist between a desire to reduce the machine's back-emf while simultaneously minimizing the machine volume (and mass) are quite apparent.

Figure 13 provides an interesting breakdown of the torque production for the best designs for each of the 6 constraint cases at rated speed. It is well known that an appealing feature of IPM synchronous machines is that their output torque 
consists of both a magnet torque component and a reluctance torque component. The sum of these two torque components should equal the rated torque value $(1 \mathrm{pu})$ for operation at the corner point speed.

As the back-emf voltage limit $V_{\text {bemf-max }}$ is reduced for Cases A through $\mathrm{D}$, the PM flux linkage decreases in proportional to the value of $V_{\text {bemf-max }}$. Therefore, it is not be surprising that the PM torque component (Fig. 13a) falls in a nearly identical fashion. Since the total torque must equal $1 \mathrm{pu}$, the reluctance torque component must increase as shown in Fig. 13b to make up for any decrease in the PM torque component. This same trend persists for Cases $\mathrm{E}$ and $\mathrm{F}$ when the value of $V_{\text {bemf-max }}$ is being held constant because the increase in the rotor diameter makes it possible to find designs with higher reluctance torque than when the rotor diameter is minimized. Under these conditions, the opportunity to reduce the magnet cost by

shifting more of the torque production burden to the reluctance torque component is attractive because of the way that the objective function has been defined.

Finally, the machine output power vs. speed envelope for the best machine in each of the six constraint cases is plotted in Fig. 14. It can be observed that all six designs meet the required power specification of delivering at least 1 pu power from the $1 \mathrm{pu}$ corner point speed to the $6 \mathrm{pu}$ maximum speed. It is interesting to note that only the machine design with the highest back-emf limit (Case A) achieves optimal flux weakening conditions as reflected in the signature monotonically increasing power vs. speed envelope that approaches a constant power value at infinite speed.

As the back-emf limit $V_{\text {bemf-max }}$ is reduced (Cases A to D), the peak power overshoot in the vicinity of the corner point speed gradually increases. This trend indicates a need to oversize the inverter [4] that is reflected in the higher required phase current at the corner point speed that was observed previously in Fig. 11a. It can also be observed that the machine designs with the lowest back-emf voltage limit (Cases D to F) barely meet the $1 \mathrm{pu}$ power requirement at maximum speed. This feature is caused by the difficulty in reducing the $d$-axis stator inductance $L_{d}$ in proportion to the reduction of the magnet flux linkage $\lambda_{p m}$ when $V_{\text {bemf-max }}$ is reduced. As a result, it becomes progressively more difficult to hold the value of the characteristic current $I_{c h}\left(=\lambda_{p m} / L_{d}\right)$ close to $1 \mathrm{pu}$ as the backemf limit is reduced from Case A to D. Unlike Case A, Cases $\mathrm{B}$ through $\mathrm{F}$ all have power vs. speed envelope shapes that are consistent with values of $I_{c h}$ that are less than the rated machine current at the corner point speed.

\section{UCG FaUlt Mode Operation}

In Sections II and III, the strategy for protecting against the UCG fault mode has been to place the entire burden on the machine design by reducing its back-emf amplitude at high speeds. In this section, attention is turned to an alternative system-level approach that shifts the burden to the inverter by designing fail-safe overvoltage protection for the inverter dc bus. It is worth noting here again that this overvoltage protection is provided naturally if the de bus is supplied by a stiff voltage source that can readily absorb regenerative power.
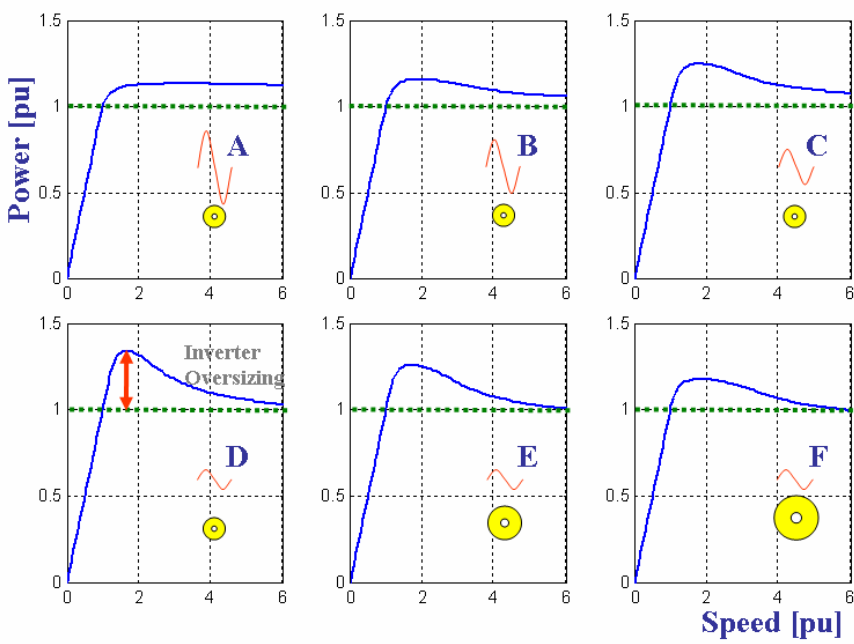

Figure 14. Power vs. speed capability envelopes for 6 optimized IPM machine designs

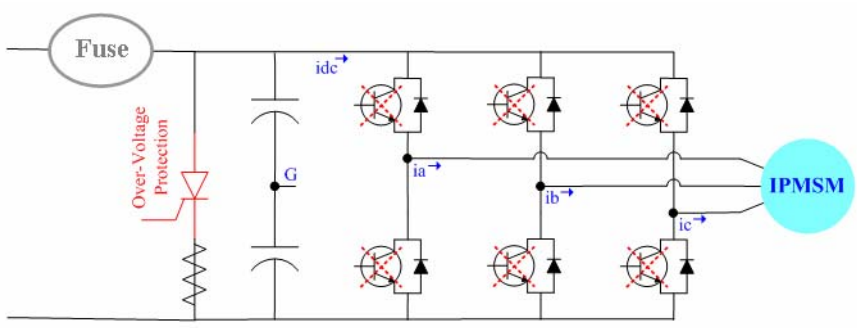

Figure 15. IPM machine drive schematic including de link crowbar circuit for UCG fault mode protection

Figure 15 provides a circuit schematic for a simple yet robust overvoltage protection scheme using a well-known thyristor-based crowbar circuit. Simulations have been carried out to predict the transient operation of the IPM machine drive during a UCG-mode fault, including operation of this crowbar circuit. It will be assumed that a sudden fault occurs during motoring operation at top speed, resulting in immediate removal of the gating signals from all of the inverter switches. This sequence of events initiates the uncontrolled generator (UCG) fault mode by which the six inverter anti-parallel diodes act as a three-phase uncontrolled rectifier bridge to deliver regenerative power from the IPM machine (acting as an alternator) back to the de bus.

Assuming that the source for the dc bus cannot accept this regenerated energy, the dc link capacitors will immediate begin to charge up towards the machine's peak line-to-line back-emf voltage (3.6pu). A voltage-threshold detection circuit will recognize that the dc link voltage is rising above its expected normal operating range and trigger the crowbar thyristor. The triggering of this thyristor provides a robust low-impedance path across the dc bus for absorbing any regenerative power delivered by the IPM machine. As shown in Fig. 15, it is assumed that a small resistance is placed in series with the crowbar thyristor in order to limit the current and absorb the energy stored in the dc bus electrolytic capacitors. It will be assumed that the value of this resistance is sufficiently low so that the dc bus is effectively short-circuited by the triggered 

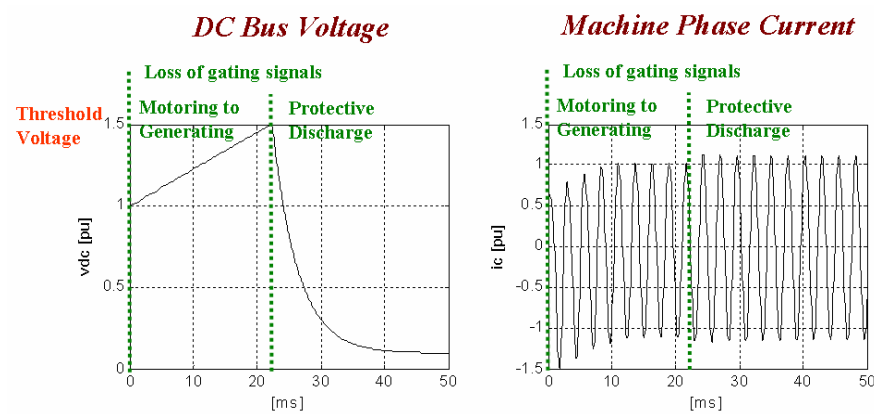

Figure 16. Simulated waveforms for the dc link voltage and one of the machine phase currents showing the UCG fault mode and protective discharge operation for the optimized Case A IPM machine

thyristor, preventing any overvoltage condition from damaging the inverter power semiconductors.

Under three-phase short circuit conditions, the phase current delivered by the IPM machine current is limited to its characteristic current $I_{c h}$ for steady-state operation. For all of the machines considered in this study for Cases A through $\mathrm{F}$, the value of $I_{c h}$ is less than or equal to the machine's rated phase current at the corner speed, ensuring that the inverter diodes will not be vulnerable to overcurrent damage after the crowbar circuit has been triggered. In order to consider worstcase conditions, the simulation has been carried out using the parameters for the Case A machine, corresponding to the highest back-emf voltage amplitude among the six cases.

In Figure 16, the resulting waveforms are provided for the dc link voltage (Fig. 16a) and for one of the phase currents (Fig. 16b) during this fault event. As noted above, the machine is delivering rated motoring power at top speed prior to removal of the inverter gating at time $t=0$. At this time instant, the dc link voltage immediately starts to rapidly increase at a rate of approximately $0.25 \mathrm{pu}$ per $10 \mathrm{~ms}$. On reaching the threshold voltage of $1.5 \mathrm{pu}$ for this simulation, the crowbar thyristor is triggered and the protective discharge of the dc bus is initiated.

It is observed in Fig. 16a that the de link voltage is quickly discharged to almost zero voltage except for a small voltage drop due to the small series crowbar resistor. The phase current waveform in Fig. 16b shows that the machine phase currents are well-behaved and safely bounded throughout the fault event.

Figure 17 provides the corresponding fault-mode waveforms for the $q$-axis current $i_{q s}$ (Fig. 17a) and the $d$-axis current $i_{d s}$ (Fig. 17b) in the rotor synchronous reference frame. The $q$-axis current is well-behaved during the motoring-togenerating transient that occurs almost immediately after $t=0$. Following triggering of the crowbar circuit, $i_{q s}$ eventually converges to nearly zero current during the protective shortcircuit operation. Similarly, the $d$-axis current (right) is bounded during the motoring-to-generating transient interval following fault initiation at $t=0$. After the crowbar circuit is triggered and the machine terminals are short-circuited, $i_{d s}$ eventually converges to the negative characteristic current $\left(-I_{c h}\right)$ which is approx. $-1.14 \mathrm{pu}$.
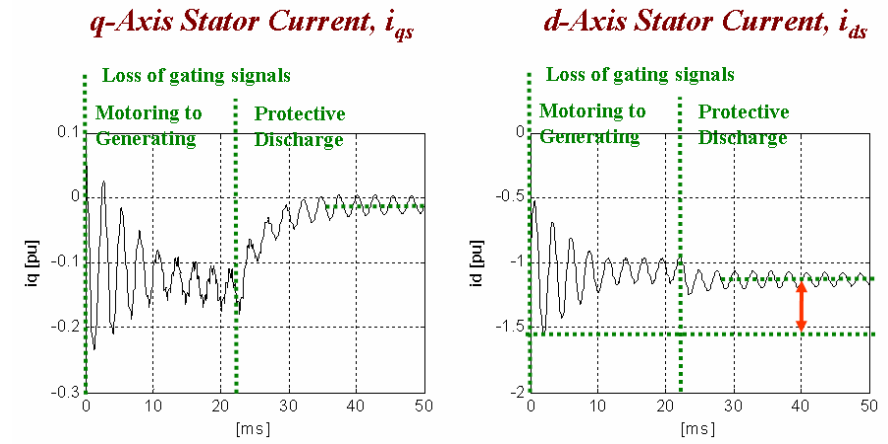

Figure 17. Simulated $q$-axis and $d$-axis current waveforms for the Case A IPM machine during the UCG fault and protection circuit response in Fig. 16

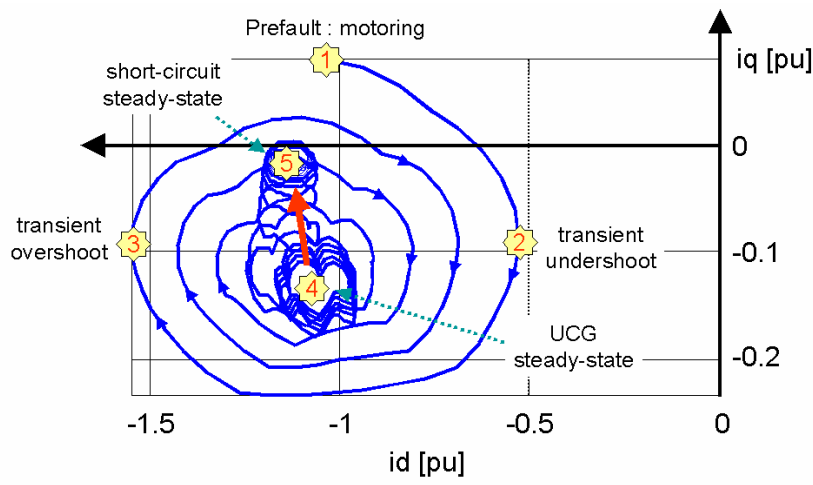

Figure 18. Simulated UCG mode current trajectory in the $d q$ current plane for the Case A IPM machine during the UCG fault and protection circuit response in Fig. 16

During the initial interval when the inverter gating is removed, the value of $i_{d s}$ transiently overshoots $-I_{c h}$ by approx. $40 \%$, reaching a peak instantaneous value slightly exceeding $-1.5 \mathrm{pu}$. Care must be taken during the design of the machine to ensure that this elevated negative $d$-axis current will not cause any bulk demagnetization of the rotor magnets.

In order to provide a useful alternative view of the phase current behavior during the UCG mode fault transient, Fig. 18 plots the $q$ - and $d$-axis current waveforms from Fig. 17 as a current trajectory in the plane of $i_{d s}$ (x-axis) and $i_{q s}$ (y-axis). Key points during the UCG fault mode transient are identified with numbers along this current trajectory. Point 1 corresponds to the pre-fault operating conditions at top speed with rated motoring power. Point 2 identifies the initial portion of the transient spiral current trajectory when the machine initially passes from motoring into generating operation. The portion of the spiral trajectory that corresponds to the highest transient demagnetizing mmf is identified as Point 3. Point 4 identifies the steady-state UCG-mode operating point that the machine begins to settle into prior to triggering of the crowbar circuit. Finally, Point 5 identifies the final steady-state operating point corresponding to $i_{d s}=-I_{c h}\left(i_{q s} \sim 0\right)$ when the terminals of the IPM machine are short-circuited while rotating at top speed.

The waveforms in Figs. 17 and 18 demonstrate that the crowbar circuit provides an effective means for protecting the inverter and the IPM machine from potentially dangerous overvoltage conditions if UCG fault-mode conditions develop at high operating speeds. This protection scheme is applicable 
to all six of the constraint cases considered in this study. The effectiveness of this overvoltage protection is not degraded even if the maximum line-to-line back-emf voltage amplitude at top speed is several times the nominal dc link voltage since the resulting short-circuit current is safely limited to $-I_{c h}$ in all cases.

\section{Conclusions}

High back-emf voltages during flux-weakening operation at elevated rotor speeds can pose serious overvoltage risks for the IPM machine and inverter if the inverter gating is suddenly removed, creating uncontrolled generator fault conditions. This paper has presented two distinct means for protecting IPM machine drives from UCG-induced damage. One of the two approaches requires that the machine be specially designed to limit its maximum back-emf voltage at top speed to fall within safe limits determined from the inverter switch ratings. The second approach introduces robust overvoltage protection into the design of the drive power electronics to ensure that the dc link voltage never reaches unsafe levels, even if the machine is designed with a maximum back-emf voltage at top speed that exceeds the inverter switch voltage ratings.

Imposing limits on the maximum back-emf voltage necessarily restricts the IPM machine's PM torque contribution, placing a higher burden on the reluctance torque component to make up the difference. Unfortunately, the resulting shift in the torque production away from magnet torque towards reluctance torque is generally unfavorable for the machine's phase current, rotor inertia, and machine volume metrics. Results presented in this paper have clearly demonstrated that reducing the maximum back-emf voltage limit penalizes the machine's phase current requirements when delivering rated power at the corner point speed. Allowing the machine volume, mass, and rotor moment of inertia to increase makes it possible to significantly reduce the phase current penalty, creating an important engineering tradeoff for the machine designer. Additional layers of rotor magnet/air cavities can be introduced into the IPM machine design in order to strengthen the reluctance torque contribution, but this will introduce further tradeoffs that involve the rotor structural integrity and fabrication costs.

However, adoption of robust overvoltage protection for the inverter $\mathrm{dc}$ bus provides a path for eliminating or at least reducing the need for back-emf voltage limits. One technique for providing this overvoltage protection using a thyristorbased crowbar circuit to short-circuit the dc bus when an incipient overvoltage condition is detected has been addressed in this paper. Under these conditions, the resulting three-phase short-circuit applied to the terminals of the IPM machine limits the steady-state machine phase current amplitude to the characteristic current. Designing the IPM machine so that its characteristic current is in the vicinity of 1 pu provides the basis for high-performance flux-weakening operation as well as safe operation following triggering of the crowbar overvoltage protection circuit. Simulation results have been used to demonstrate the smooth transition associated with the initiation of the UCG fault mode followed by triggering of the crowbar overvoltage protection circuit.

The study results presented in this paper were derived for a particular IPM machine configuration with two magnet layers per pole and a specific set of performance requirements. Despite these details, all available evidence suggests that the major conclusions presented in this paper regarding the design tradeoffs resulting from the imposition of back-emf voltage limits apply rather broadly to IPM machines with different configurations and performance specifications.

In conclusion, IPM machine drive designers faced with the task of protecting the drive system from UCG-induced overvoltage conditions are advised to carefully consider all options available to them. Although the imposition of limits on the maximum back-emf voltage provides a conservative solution that may be acceptable in some applications, the resulting penalties on key performance metrics may prove to be highly undesirable. Under such conditions, the system-level approach based on the introduction of robust overvoltage protection for the dc bus deserves serious consideration as an effective alternative solution that avoids the necessity of costly machine design penalties.

\section{REFERENCES}

[1] A. Kabasawa, K. Takahashi, "Development of the IMA Motor for the V6 Hybrid Midsize Sedan”, SAE Paper 05P-150, 2004.

[2] T.J.E. Miller, Brushless Permanent-Magnet and Reluctance Motor Drives, Clarendon Press, Oxford, 1989.

[3] A. Adnanes, R. Nilssen, and R. Rad, "Power Feed-Back during Controller Failure in Inverter Fed Permanent Magnet Synchronous Motor Drives with Flux Weakening", in Proc. of 1992 IEEE Power Elec. Spec. Conf. (PESC), pp. 958-963, 1992.

[4] T.M. Jahns, "Component Rating Requirements for Wide Constant Power Operation of Interior PM Synchronous Machine Drives", in Rec. of 2000 IEEE Industry Applications Society Annual Meeting, vol. 3, pp. 16971704, October 2000.

[5] A.M. EL-Refaie, and T.M. Jahns, "Comparison of Synchronous PM Machine Types for Wide Constant-Power Speed Range Operation", in Rec. of 2005 IEEE Industry Applications Society Annual Meeting, Hong Kong, October 2005, CD-ROM.

[6] E.C. Lovelace, "Optimization of a Magnetically Saturable Interior PM Synchronous Machine Drive", PhD Thesis, Dept. of Elec. Eng. \& Comp. Sci., MIT, 2000.

[7] Damir Zarko, "A Systematic Approach to Optimized Design of Permanent Magnet Motors with Reduced Torque Pulsations", PhD Thesis, Dept. of Elec. \& Comp. Eng., UW-Madison, 2004. 